\title{
Impact of Comorbidities on the Prognosis of Pediatric Postural Tachycardia Syndrome
}

\author{
Yaru Wang' \\ Junbao $\mathrm{Du}^{1,2}$ \\ Xueying $\mathrm{Li}^{3}$ \\ Ping Liu' \\ Yuli Wang' \\ Ying Liao' \\ Hongfang Jin' \\ 'Department of Pediatrics, Peking \\ University First Hospital, Beijing, 100034, \\ People's Republic of China; ${ }^{2}$ Key Laboratory \\ of Molecular Cardiovascular Sciences, \\ Ministry of Education, Beijing, 100034, \\ People's Republic of China; ${ }^{3}$ Department of \\ Medical Statistics, Peking University First \\ Hospital, Beijing, 100034, People's Republic \\ of China
}

Objective: To investigate the influence of comorbidities on the prognosis of pediatric postural tachycardia syndrome (POTS).

Methods: In this retrospective cohort study, 275 children with POTS admitted to the Department of Pediatrics at Peking University First Hospital were recruited from 2016 to 2019 and followed up. The participants were divided into simple POTS (S-POTS, n=156 cases) and POTS with comorbidities (Co-POTS, $n=119$ cases) groups according to whether they were complicated with comorbidities. A Cox regression analysis was used to identify the prognostic risk factors for children with POTS, while Kaplan-Meier curves were applied to compare the cumulative symptom remission rate (CSRR) between the two groups. The rehospitalization of the children between the two groups was also compared to explore the influence of comorbidities.

Results: Twenty-one participants (7.6\%) were lost during a median follow-up of 24 months. The Cox regression model showed that comorbidities and body mass index (BMI) were associated with the CSRR of the children with POTS. The CSRR of pediatric POTS alone was 1.748 times higher than that of patients with comorbidities, and the CSRR was decreased by $5.1 \%$ for each $1 \mathrm{~kg} / \mathrm{m}^{2}$ increase in BMI. The most common comorbidity in children with POTS in this study was allergic disorders, followed by the psychological diseases. The patients in the CoPOTS group had a lower CSRR than those in the S-POTS group ( $\log$ rank $\mathrm{P}=0.0001)$. In addition, compared with those of the S-POTS group, the total number of rehospitalizations was high $(\mathrm{P}=0.001)$, and the total hospital stays were long in the Co-POTS group $(\mathrm{P}<0.001)$.

Conclusion: Complicating with comorbidities, pediatric patients with POTS had lower CSRR and more rehospitalizations than those without comorbidities. More attention should be given to comorbidities when managing pediatric POTS.

Keywords: postural tachycardia syndrome, comorbidity, allergic disorders, prognosis, children

\section{Introduction}

Postural tachycardia syndrome (POTS) is a group of disorders featuring symptoms of orthostatic intolerance (OI) (including dizziness, headache, nausea, sweating, chest distress, palpitations, blurred vision, etc.) and significantly increased heart rates when standing. It is a common cause of $\mathrm{OI}^{1}$ in children, and the symptoms can seriously affect the patients' quality of life. ${ }^{2}$

Roma et al conducted a questionnaire survey on the prognosis of children with POTS and found that symptoms in $86 \%$ of the participants were improved or relieved or became intermittent an average of 5 years after the initial treatment. ${ }^{3}$ Tao et al followed up a group of children with POTS for 6 years
Correspondence: Ying Liao; Hongfang lin Department of Pediatrics, Peking University First Hospital, Beijing, I00034, People's Republic of China Tel +8610-83573238

Fax +8610-66530532

Email liaoyingwww@163.com;

jinhongfang5I@I26.com 
and showed a cumulative asymptomatic rate of $85.6 \%{ }^{4}$ These above reports suggest that the prognosis of pediatric POTS is generally benign. At present, the reported comorbidities in children with POTS mainly include allergic disorders, migraine, psychological disorders, hyperventilation syndrome, chronic fatigue syndrome, hypermobile Ehlers-Danlos syndrome and hypermobility spectrum disorder (hEDS/HSD), gastrointestinal dysfunction, and fibromyalgia. ${ }^{5-13}$ A study from the United States (US) reported that the spectrum of comorbidities in children with POTS mainly included sleeping disorders, gastrointestinal dysfunction, and headache. ${ }^{14}$ The above comorbidities may be related to the mechanisms underlying POTS, such as autonomic nervous dysfunction ${ }^{15}$ and vascular dysfunction, ${ }^{16}$ which may theoretically affect the clinical manifestations and prognosis of children with POTS. Investigating the impact of comorbidities on the prognosis of POTS in children would significantly deepen the understanding of the outcome of POTS and help to implement a more effective strategy in patient management. However, the influence of comorbidities on the outcome of pediatric POTS has not been identified. Therefore, this study was performed to explore the influence of comorbidities on the prognosis of children with POTS in our center.

\section{Methods}

\section{Study Subjects}

Our retrospective cohort study enrolled hospitalized children with a diagnosis of POTS in the Department of Pediatrics in Peking University First Hospital from January 2016 to December 2019. The patients' clinical information, including age of onset, sex, age at admission, body mass index (BMI), course of the disease before admission, frequency of symptoms of OI, supine heart rate, maximum upright heart rate, supine blood pressure, therapeutic methods, and comorbidities, was collected according to the data from the Beijing Kaihua Medical Recording Management Digital System. This study was approved by the Ethics Committee of Peking University First Hospital (2021-095) and was conducted in accordance with the Declaration of Helsinki. Since it was a retrospective study, verbal consent was obtained from the parents of the subjects over the telephone recorded by a professional investigator before the follow-up. The verbal consent protocol was approved by the Ethics Committee of Peking University First Hospital.
The inclusion criteria were as follows: (1) patients aged 5-18 years old; (2) hospitalized patients with a diagnosis of POTS in the Department of Pediatrics in Peking University First Hospital from 2016 to 2019; and (3) patients diagnosed with or without comorbidities, including functional gastrointestinal disorders (including periodic vomiting syndrome and functional abdominal pain), pediatric psychological disorders (including psychosomatic illness, anxiety disorder, mood disorders, and sleeping disorders), hyperventilation syndrome, chronic fatigue syndrome, allergic disorders (including allergic rhinitis, food allergy, adverse reactions to drugs, childhood asthma, atopic eczema, and urticaria), migraine, hEDS/HSD, and fibromyalgia. ${ }^{5-13}$ The following patients were excluded: (1) those with incomplete basic clinical data; (2) children with a suspected diagnosis instead of a confirmed diagnosis; (3) children complicated with epilepsy, cardiogenic syncope, or hypertension; and (4) those with course of the disease less than 1 month.

In this study, the included participants were divided into a simple POTS (S-POTS) group and a POTS with comorbidity (Co-POTS) group according to whether they were complicated with comorbidities.

\section{Diagnostic Criteria of Pediatric POTS}

The diagnosis of pediatric POTS followed the corresponding guidelines for children. ${ }^{17}$ That is, the child suffers from typical symptoms of OI, and during the standing test or head-up tilt test (HUTT), the upright heart rate increases by $\geq 40$ beats $/ \mathrm{min}$ within 10 minutes or the peak upright heart rate meets the following criteria $(\geq 130$ times/min for children aged 6-12 years old; $\geq 125$ times/ min for children aged 13-18 years old). At the same time, conditions such as cardiovascular disease, metabolic disease, or neurological disease were also excluded.

\section{Symptom Score (SS) for Children with POTS}

SS of OI was used to assess the severity of pediatric POTS symptoms. ${ }^{18}$ Symptoms of OI mainly included dizziness, nausea, palpitations, headache, chest tightness, syncope, hand tremors, blurred vision, sweating, and inattention. For each symptom mentioned above, 0 score indicates that no such symptom occurred; 1 score indicates that the frequency of the symptom is once per month; 2 scores indicate a frequency of 2-4 times per month; 3 scores indicate a frequency of 2-7 times per week; and 4 scores indicate a frequency of at least 
once a day. The total symptom score of one patient is the sum of scores calculated from all the symptoms.

\section{Diagnostic Criteria of Comorbidities}

The data of the above comorbidities including allergic diseases, ${ }^{19}$ migraine, ${ }^{20}$ functional gastrointestinal disorders, ${ }^{21}$ chronic fatigue syndrome, ${ }^{22}$ joint hypermobility syndrome,${ }^{23}$ psychological disorders,${ }^{24}$ fibromyalgia, ${ }^{25}$ hyperventilation syndrome, ${ }^{26}$ and Ehlers-Danlos syndromes $(E D S)^{27}$ referred to the listed diagnosis at the participants' discharge. The diagnosis of comorbidities was made by corresponding specialists through clinical consultations and according to acknowledged diagnostic criteria $^{19-27}$ during the participants' hospitalization.

\section{Follow-Up}

The follow-up of each patient began with the first discharge from the hospital. The patients were followed up either by outpatient visits or by telephone calls carried out by specialized investigators. During the follow-up, the investigators evaluated the symptom control of the patients. The last time point of follow-up was April 2021. The follow-up data were recorded and organized in the follow-up database by a designated investigator and were verified by another researcher. In this study, symptom remission was recognized as the end point event, which was defined as an asymptomatic status lasting for at least 3 consecutive months and no recurrence until the last follow-up.

\section{Statistical Analysis}

Statistical processing of the data was conducted in SPSS 25.0 (IBM, Armonk, New York) and GraphPad 8.0 software (GraphPad Software, San Diego). The normality was examined by the single-sample Kolmogorov-Smirnov method. The measurement data that displayed a normal distribution are presented as the mean \pm standard deviation, and $t$-test was used to test for differences; otherwise, the data are presented as the median $\left(\mathrm{P}_{25}, \mathrm{P}_{75}\right)$ and were compared by the Wilcoxon rank sum test. The enumeration data are described by rate (\%) or numbers, and the $\chi^{2}$ test was used for difference analysis. Results with $\mathrm{P}<0.05$ were perceived as remarkable differences. Univariate analysis of a Cox regression risk model was conducted using the Enter method, and the parameters with $\mathrm{P}<0.1$ were introduced into the multivariate analysis. Spearman correlation analysis was used to assess whether there was a correlation between the two variables. Finally, uncorrelated variables were put into the multivariate analysis using the conditional forward method. Kaplan-Meier (K-M) curves were used to compare the cumulative symptom remission rate (CSRR) of patients between the S-POTS and Co-POTS groups, with symptom remission being the end point during the follow-up. The end point event was defined as " 1 ". K-M curves were compared using the Log rank test, and the results with $\mathrm{P}<0.05$ were perceived as remarkable differences. The median followup duration of pediatric POTS was calculated using the reverse K-M method.

\section{Results}

\section{Patients Population}

A total of 364 pediatric patients were diagnosed with POTS between January 2016 and December 2019 in our center, and 89 of them were excluded according to the inclusion and exclusion criteria. The remaining 275 patients were enrolled and followed up, of whom 21 (7.6\%) were lost because the patients did not return to the outpatient department or refused telephone follow-up. The flow chart of the screening process is shown in Figure 1. The median age of the 275 patients was 12.0 (10.0, 14.0) years old, including 117 males and 158 females. The median and maximum follow-up durations were 24 months and 59 months, respectively. The 275 patients were separated into the S-POTS group consisting of 156 patients (62 males and 94 females) with a median age of $12.0(10.0,13.7)$ years and the Co-POTS group consisting of 119 patients (55 males and 64 females) with a median age of $12.0(10.0,14.0)$ years. No significant difference was found in the sex ratio, age at admission, age at onset, BMI, course of the disease before admission, SS at admission, supine heart rate, supine blood pressure, maximum upright heart rate, or therapeutic approaches between the two groups, as shown in Table 1.

\section{Cox Regression Analysis of Prognosis in Children with POTS}

In the Cox regression model, sex, age at admission, age at onset, BMI, course of the disease before admission, SS at admission, supine heart rate, supine systolic and diastolic blood pressure, maximum upright heart rate, therapeutic methods, and comorbidities were taken as independent variables, and the CSRR was taken as the dependent variable. Univariate Cox regression analysis indicated that prognostic risk factors for children with POTS were comorbidities $[\mathrm{P}=0.001$, hazard 


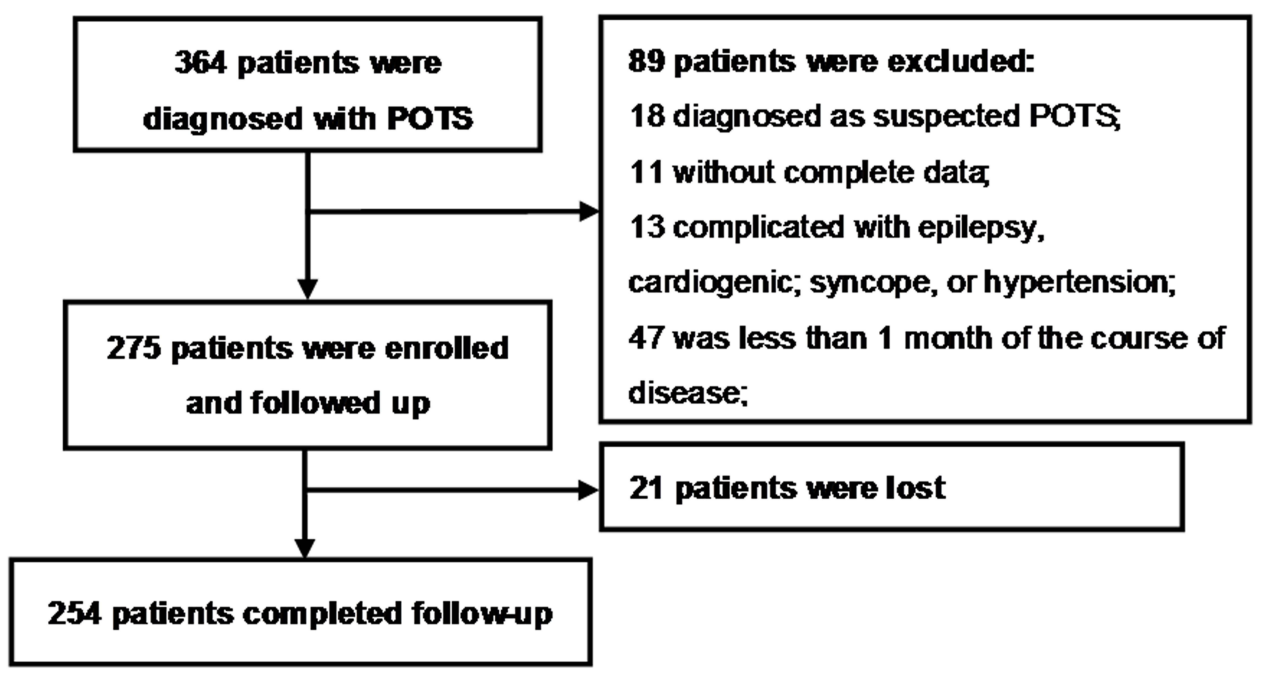

Figure I Flow-chart of patient inclusion. Of 364 patients with POTS, 89 were excluded according to the exclusion criteria, and the remaining 275 patients were followed up. Finally, 254 patients completed follow-up.

Abbreviation: POTS, postural tachycardia syndrome.

ratio (HR): $1.759,95 \%$ confidence interval (CI): 1.274-2.428] and high baseline BMI ( $\mathrm{P}=0.036$, HR: 0.948, 95\% CI: $0.902-$ 0.997), as shown in Figure $2 \mathrm{~A}$.

The variables with $\mathrm{P}<0.1$ in the univariate Cox regression model (comorbidities and BMI) were introduced into the multivariate Cox regression model, and no correlation was determined between comorbidities and BMI ( $\mathrm{r}=0.014$, $\mathrm{P}=0.812$ ). The results of multivariate Cox regression analysis suggested that comorbidities and high BMI were both independent factors that adversely affected the symptom remission rate of children with POTS. Specifically, the CSRR of pediatric POTS alone was 1.748 times higher than that of the patients with comorbidities ( $\mathrm{P}=0.001$, HR: $1.748,95 \% \mathrm{CI}$ : 1.266-2.413), and for each $1 \mathrm{~kg} / \mathrm{m}^{2}$ increase in baseline BMI of the patients, the CSRR was decreased by $5.1 \%(\mathrm{P}=0.042$, HR: 0.949, 95\% CI: 0.902-0.998), as shown in Figure 2B.

\section{Spectrum of Comorbidities in Children with POTS}

In total, 43.3\% (119/275) of children with POTS were complicated with comorbidities, among whom 35.6\% (98/275) were diagnosed with 1 comorbidity, $6.9 \%$ (19/275) with 2 comorbidities, and $0.8 \%(2 / 275)$ with 3 and 4 comorbidities, as shown in Figure 3A. The comorbidities mainly included

Table I Clinical Characteristics of Patients in the S-POTS Group and Co-POTS Group

\begin{tabular}{|l|l|l|l|l|}
\hline Items & S-POTS Group & Co-POTS Group & Z/t/ $\chi^{2}$ & P value \\
\hline Number & 156 & 119 & & \\
Sex (male/female) & $62 / 94$ & $55 / 64$ & 1.158 & 0.282 \\
Age at admission, years old & $12.0(10.0,13.7)$ & $12.0(10.0,14.0)$ & -0.672 & 0.502 \\
Age of onset, years old & $11.0(8.0,12.0)$ & $11.0(7.0,13.0)$ & -0.451 & 0.652 \\
BMI, kg/m & $18.3(16.2,20.6)$ & $17.9(16.2,20.0)$ & -0.238 & 0.812 \\
Course of disease before admission, month & $12.0(3.0,24.0)$ & $12.0(4.0,36.0)$ & -0.588 & 0.556 \\
Symptom score at admission & $5.0(3.0,8.0)$ & $6.0(4.0,8.0)$ & -1.749 & 0.080 \\
Supine heart rate, bpm & $76.4 \pm 9.8$ & $75.1 \pm 9.1$ & 1.121 & 0.263 \\
Supine systolic pressure, mmHg & $107.0(99.0,115.0)$ & $106.0(99.0,112.0)$ & -0.903 & 0.367 \\
Supine diastolic pressure, mmHg & $61.0(56.0,66.0)$ & $62.0(57.0,67.0)$ & -0.615 & 0.539 \\
*Maximum upright heart rate, bpm & $122.3 \pm 11.6$ & $121.8 \pm 13.1$ & 0.354 & 0.724 \\
\#Treatment (medication/nonmedication) & $78 / 78$ & $56 / 63$ & 0.234 & 0.629 \\
\hline
\end{tabular}

Notes: Values are mean \pm standard deviation, median $\left(\mathrm{P}_{25}, \mathrm{P}_{75}\right)$, or number; ${ }^{\#}$ Nonmedication therapy included health education, upright training, or oral rehydration salts; Medication therapy included midodrine or metoprolol on the basis of nonmedication therapy; *The maximum upright heart rate refers to the peak heart rate within the initial 10 minutes when the patient stands up during the standing test or when the patient is tilted during the HUTT at his or her first hospitalization.

Abbreviations: BMI, body mass index; bpm, beat per minute; S-POTS, simple postural tachycardia syndrome; Co-POTS, postural tachycardia syndrome with comorbidity. 


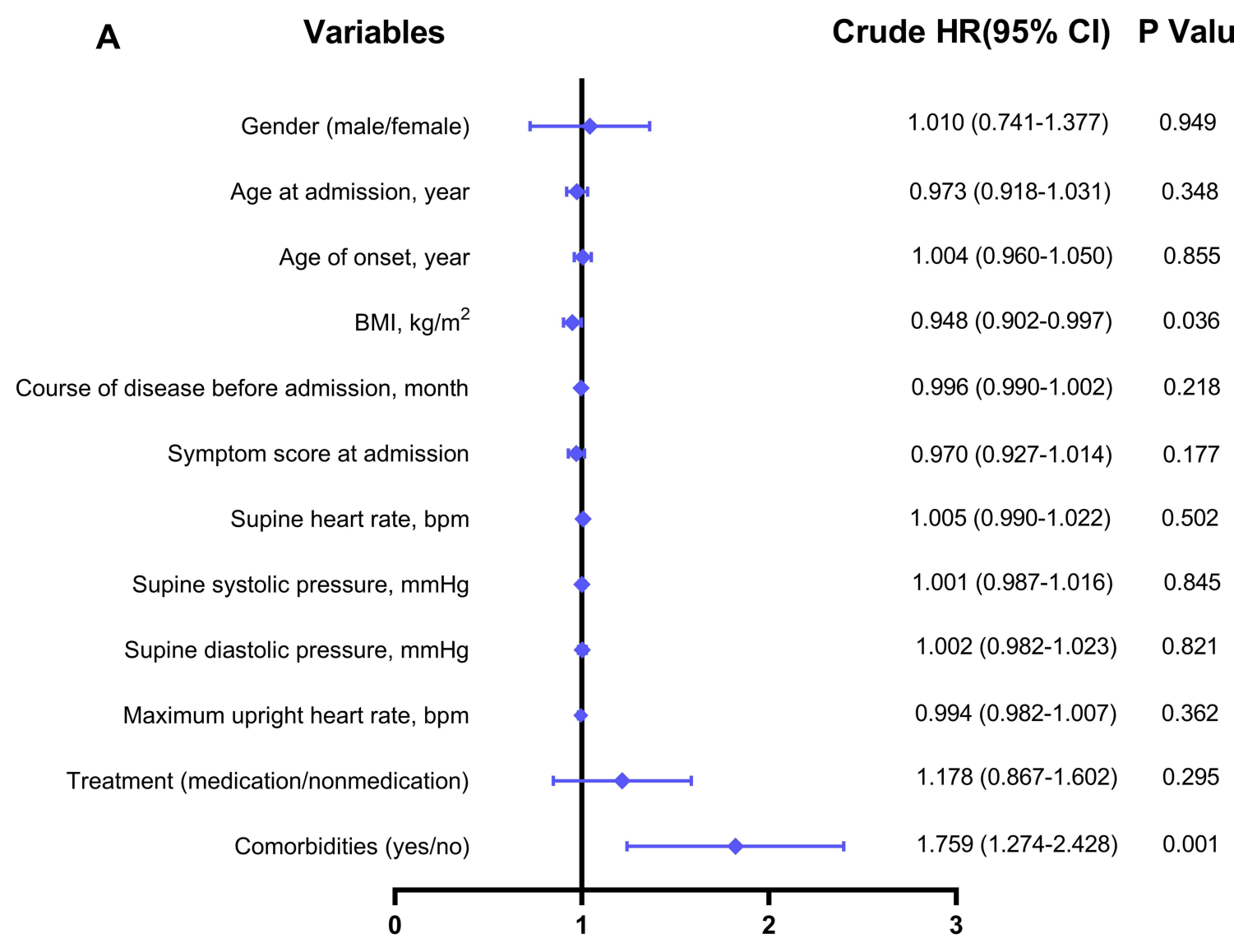

B Variables

Adjusted HR(95\% Cl) P Value

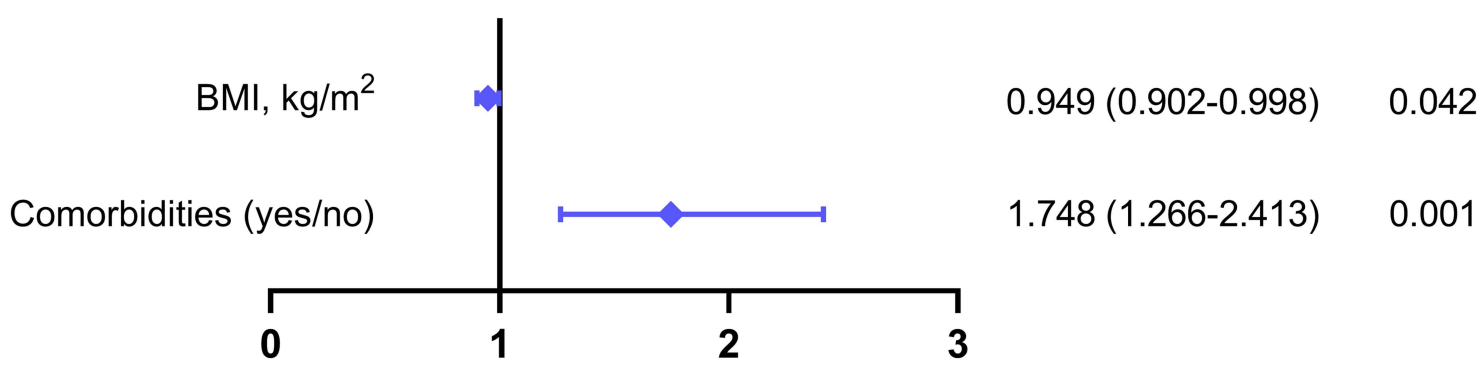

Figure 2 Forest plot for the outcomes of univariate and multivariate Cox regression model analysis on risk factors for prognosis in children with POTS. (A) Forest plot presenting the crude hazard ratio for the outcomes of univariate Cox regression model analysis. (B) Forest plot presenting the adjusted hazard ratio for the outcomes of multivariate Cox regression model analysis. Each blue rhombus represents the $\mathrm{HR}$ value of the corresponding variable, and the intersecting blue transverse line represents the $95 \% \mathrm{Cl}$. Abbreviations: POTS, postural tachycardia syndrome; BMI, body mass index; bpm, beat per minute; $\mathrm{HR}$, hazard ratio; $\mathrm{Cl}$, confidence interval.

allergic disorders, psychological disorders, migraine, hyperventilation syndrome, and chronic fatigue syndrome. The distribution of comorbidities is shown in Figure 3B. Allergic disorders were the most common comorbidities, accounting for $83.2 \%$ (99/119), among which allergic rhinitis was the most common. The second most common entity was psychological disorders, accounting for $16.0 \%$ of comorbidities (19/ 119), as shown in Tables 2 and 3.

\section{Influence of Comorbidities on the} Prognosis of Children with POTS

A total of 156 cases were collected in the S-POTS group, 11 of which were lost to follow-up, and 119 cases were collected in the Co-POTS group, 10 of which were lost to follow-up. As shown in Figure 4, the results of $\mathrm{K}-\mathrm{M}$ survival analysis suggested that the CSRR of patients 
A

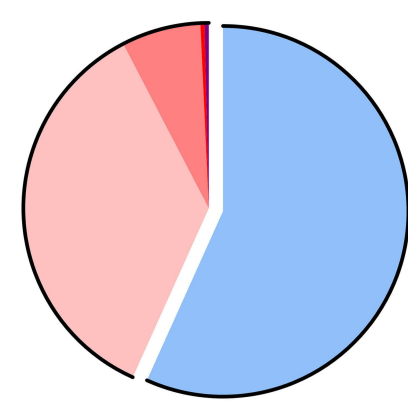

Total number $=275$
S-POTS group, N=156, 56.7\%

$\square$ Co-POTS group: 1 comorbidity, N=98, 35.6\%

Co-POTS group: 2 comorbidities, $\mathrm{N}=19,6.9 \%$

$\rightarrow$ Co-POTS group: 3 comorbidities, $\mathrm{N}=1,0.4 \%$

Co-POTS group: 4 comorbidities, $\mathrm{N}=1,0.4 \%$

B

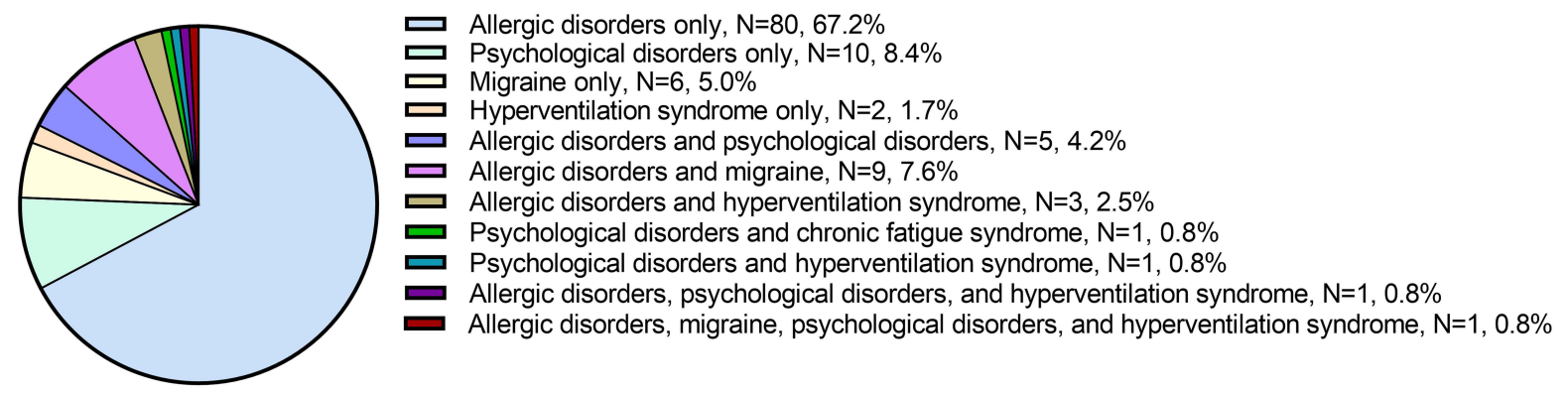

Total number of Co-POTS group $=119$

Figure 3 The spectrum of comorbidities in children with POTS. (A) The proportion of patients complicated or not complicated with comorbidities. (B) The proportion of different comorbidities of patients in the Co-POTS group.

Abbreviations: POTS, postural tachycardia syndrome; S-POTS, simple POTS; Co-POTS, POTS with comorbidity.

in the S-POTS group was statistically higher than that in the Co-POTS group ( $\log$ rank $\mathrm{P}=0.0001)$. The median symptom remission times of the S-POTS group and the Co-POTS group were 9 months and 30 months, respectively.

Table 2 The Spectrum of Allergic Disorders in Comorbidities of Pediatric POTS

\begin{tabular}{|l|l|l|}
\hline Items & $\begin{array}{l}\text { Number of } \\
\text { Participants } \\
\text { with Different } \\
\text { Comorbidities }\end{array}$ & $\begin{array}{l}\text { Percentage of } \\
\text { the Number of } \\
\text { Comorbidities } \\
\text { (N=1 I 19, \%) }\end{array}$ \\
\hline Allergic disorders & 99 & $83.2 \%$ \\
Allergic rhinitis & 24 & $20.2 \%$ \\
Food allergy & 18 & $15.1 \%$ \\
Adverse reactions to drugs & 14 & $11.8 \%$ \\
Childhood asthma & 5 & $4.2 \%$ \\
Atopic eczema & 7 & $5.9 \%$ \\
Urticaria & 3 & $2.5 \%$ \\
\#Multiple allergic disorders & 28 & $23.5 \%$ \\
\hline
\end{tabular}

Notes: ${ }^{\#}$ Complicated with more than one allergic disorders, among them, 18 cases complicated with 2 allergic disorders, 8 cases complicated with 3 allergic disorders, and 2 cases complicated with 4 allergic disorders.

Abbreviation: POTS, postural tachycardia syndrome.

\section{Burden of Rehospitalization of Patients in the S-POTS Group and Co-POTS Group}

As shown in Table 4, the patients in the Co-POTS group had more total hospitalizations $(\mathrm{P}=0.001)$ and longer total hospital stays $(\mathrm{P}<0.001)$ than those in the S-POTS group.

\section{Discussion}

The comorbidities of POTS have attracted increasing attention in recent years, but the influence of comorbidities on the prognosis of children with POTS remains unclear. This single-center retrospective cohort study first revealed that comorbidities can negatively influence the prognosis of children with POTS. In this study, Cox regression model analysis showed that comorbidities and high BMI were independent factors that adversely affected the symptom remission rate of pediatric POTS. Of note, the CSRR of patients in the S-POTS group was 1.748 times that of the Co-POTS group. Comorbidities significantly increase the rehospitalization burden of pediatric patients with POTS.

A previous study reported that the incidence of allergic disorders in children with POTS was $41 \%$ in Chinese patients, ${ }^{5}$ while a cross-sectional survey from over 15 
Table 3 The Spectrum of Psychological Disorders in Comorbidities of Pediatric POTS

\begin{tabular}{|l|l|l|}
\hline Items & $\begin{array}{l}\text { Number of Participants with Different } \\
\text { Comorbidities }\end{array}$ & $\begin{array}{l}\text { Percentage of the Number of } \\
\text { Comorbidities (N=I I 9, \%) }\end{array}$ \\
\hline Psychological disorders & 19 & $16.0 \%$ \\
Psychosomatic illness & $\mathrm{I}$ & $0.8 \%$ \\
Conversion disorder & $\mathrm{I}$ & $0.8 \%$ \\
Anxiety disorder & $\mathrm{I}$ & $0.8 \%$ \\
Mood disorders & 9 & $7.6 \%$ \\
Depression & 3 & $2.5 \%$ \\
Unspecified mood disorders & 6 & $5.0 \%$ \\
Sleeping disorder & $\mathrm{I}$ & $0.8 \%$ \\
Sleeping disorder and anxiety disorder & 3 & $2.5 \%$ \\
Anxiety disorder and mood disorder & 3 & $2.5 \%$ \\
Psychosomatic illness and mood disorder & $\mathrm{I}$ & $0.8 \%$ \\
\hline
\end{tabular}

Abbreviation: POTS, postural tachycardia syndrome.

countries found that $20 \%$ of children with POTS were complicated with asthma. ${ }^{28}$ This study reported the spectrum of comorbidities among children with POTS, among which allergic disorders were the main comorbidities, accounting for $36.0 \%$ of the total patients, followed by psychological disorders, accounting for $6.9 \%$ of the total patients. In addition, migraine, hyperventilation syndrome, and chronic fatigue syndrome were also included as comorbidities.

The mechanisms by which comorbidities are related to the symptom remission rate of pediatric POTS are not completely clear. Chelimsky's results suggested that the

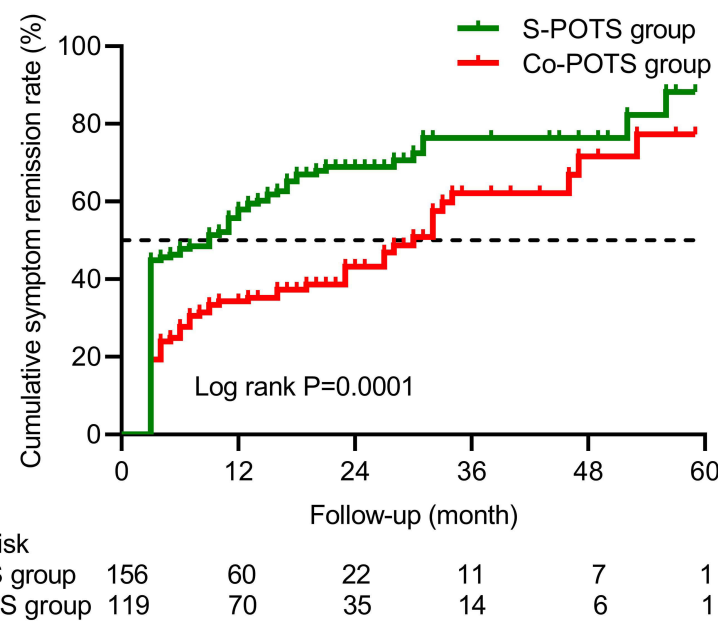

Figure 4 Influence of comorbidities on prognosis of pediatric POTS. The K-M curves showed the cumulative symptom remission rate in the S-POTS and CoPOTS groups during follow-up. The green curve and red curve represent the cumulative symptom remission rate for the S-POTS group and Co-POTS group, respectively.

Abbreviations: K-M, Kaplan-Meier; POTS, postural tachycardia syndrome; S-POTS, simple POTS; Co-POTS, POTS with comorbidity. relationship between them needed further studies. ${ }^{29}$ According to the current points of view, relative central hypovolemia, autonomic nervous dysfunction, and peripheral vascular dysfunction are speculated to be the mechanisms for POTS, $^{30}$ which may be enhanced by complications with comorbidities.

Allergic disorders were the most common comorbidities in children with POTS in this study, and it is speculated that the mechanisms for the comorbidity of allergic disorders and POTS may be related to excessive vasodilation. One of the classical mechanisms for allergy is type I hypersensitivity, which may result in mast cell degranulation and subsequent release of vasoactive substances, such as leukotrienes, histamines, and prostaglandins. ${ }^{16}$ Furthermore, mast cell activation disorder (MCAD), a condition in which mast cells increase in number or excessively release inflammatory mediators, ${ }^{31}$ was considered to be a comorbidity of adult POTS, and some of which might be secondary to allergic disorders. ${ }^{32,33}$ A previous study on adults reported that patients with POTS were complicated with abnormal mast cell activation and significant increases in methylhistamine, leukotriene, and prostaglandin $\mathrm{D}_{2}$ in their urine. ${ }^{34}$ These inflammatory vasoactive substances may aggravate the dilation of peripheral blood vessels and increase vasopermeability, which can theoretically facilitate the occurrence of OI.

The second most common comorbidity in this study was psychological disorders. Psychological factors can not only amplify the objective discomfort resulting from OI but also add the diversification and complexity of the clinical manifestation in pediatric POTS. Meanwhile, 
Table 4 Evaluation of Rehospitalization Burden in Children with POTS

\begin{tabular}{|l|c|c|c|c|}
\hline Items & S-POTS Group & Co-POTS Group & Z & P value \\
\hline Total number of rehospitalizations (time) & $1.0(1.0,2.0)$ & $1.0(1.0,2.0)$ & -3.368 & 0.001 \\
Total hospital stay (day) & $8.0(7.0,12.0)$ & $10.0(7.0,17.0)$ & -3.593 & $<0.001$ \\
\hline
\end{tabular}

Notes: Values are median $\left(\mathrm{P}_{25}, \mathrm{P}_{75}\right)$.

Abbreviations: POTS, postural tachycardia syndrome; S-POTS, simple POTS; Co-POTS, POTS with comorbidity.

physical discomfort and frustrations in school and daily life may in turn promote the development of psychological disorders. Consequently, it is reasonable to speculate that psychological disorders and POTS may interact with each other, further aggravating the symptoms of patients and increasing the difficulty in their management.

Migraine and hyperventilation syndrome, which were also common comorbidities of pediatric POTS in our study, are thought to be related to autonomic nervous dysfunction. A previous study suggested that children with migraine may have a genetic susceptibility to autonomic dysfunction, ${ }^{35}$ while it has been reported that patients with hyperventilation syndrome are prone to sympathetic excitation. ${ }^{36}$ The imbalance of the autonomic nervous system may play a vital role in the development of POTS. It is believed that, headache, as a common symptom of pediatric POTS, should be carefully investigated. Ojha et al reported that $45.5 \%$ of pediatric POTS patients complained of headache. ${ }^{14}$ The percentage of patients with headache in our study $(96 / 275,34.9 \%)$ was similar to their study. In addition, the proportion of patients with headache in the Co-POTS group was remarkably higher than that in the S-POTS group (54/65 vs 42 / 114; $\chi^{2}=10.118 ; \mathrm{P}=0.001$ ). Migraine that is sometimes characterized by recurrent headache, should be paid more attention to.

Sleeping disorders in children with POTS have attracted increasing attention. Like other behavioral problems in children, abnormal sleep is usually defined by parental observations. We reviewed all the medical records of the patients in our study and found that $10.2 \%(28 / 275)$ of the children patients used to suffer from abnormal sleeping quantity or poor sleeping quality according to the history offered by themselves and their parents. The proportion of the patients with self-reported poor sleeping was significantly higher in the Co-POTS group than that in the S-POTS group $\left(18 / 101\right.$ vs $\left.10 / 146 ; \chi^{2}=5.607 ; \mathrm{P}=0.018\right)$. Since the patients with POTS in our cohort did not regularly undergo sleeping studies, the sleeping disorders may be under-diagnosed. The interaction between sleeping disorders and POTS is not clear. Some studies reported that a sleep duration less than 8 hours is a risk factor for pediatric POTS and that sleep promotion therapy is an effective way to treat POTS in children. ${ }^{37,38}$ Although some clues may be found in a series of endocrine disturbances caused by insufficient sleep, ${ }^{39,40}$ further investigation is needed to elucidate the mechanism.

One of the possible explanations for the comorbidity with POTS may be immune mechanisms. OI is one of the common clinical manifestations of chronic fatigue syndrome. It can be speculated from previous studies that autonomic nervous dysfunction in patients diagnosed with comorbidities of POTS and chronic fatigue syndrome may be related to autoantibodies against G-proteincoupled receptors and small fiber neuropathy. ${ }^{41-43}$ However, at present, the exact role of these antibodies is not fully understood.

The co-existence of POTS and hEDS/HSD in adults was reported. ${ }^{44}$ The findings revealed that $22.7 \%$ of pediatric POTS had EDS and $39.0 \%$ had hypermobility spectrum disorder (HSD). ${ }^{45}$ Another study demonstrated that HSD accounted for $36.3 \%$ and EDS accounted for $20.0 \%$ among the POTS patients aged under 19 years old. ${ }^{46}$ However, data are not available in Chinese children. Furthermore, the triad of POTS-MCAS-EDS should be paid more attention to. ${ }^{33,34}$ This syndrome was not reported in this study, and the missed diagnosis may be related to the single center and small number of the cases.

In addition, the CSRR of three comorbidity subgroups (allergic disorders, psychological disease, and others) was also compared by K-M curves, revealing no differences among the three subgroups. However, further studies including more cases are still needed to clarify the influence of each type of comorbidity on the prognosis of pediatric POTS. We also found that $7.6 \%$ of the participants in this study had 2 or more comorbidities. However, more cases are still needed to further investigate the impacts of multiple comorbidities on POTS.

In addition to comorbidities, BMI was another factor related to the symptom remission rate of POTS in this 
study. Specifically, patients with higher BMI were more likely to demonstrate lower CSRR. Some clues in previous studies may help to explain this result. Stewart's study showed a positive correlation between BMI and blood volume, suggesting that patients with a lower BMI usually have lower blood volume. ${ }^{47}$ One study published in 2016 showed that the therapeutic efficacy of oral rehydration salt for POTS children with low BMI was usually better. ${ }^{48}$ Therefore, patients with low BMI are likely to benefit from increased salt and water intake and achieve a higher symptom remission rate.

Exploring the influence of comorbidities on the prognosis of pediatric POTS possesses important value in the understanding and management of pediatric POTS. The results of this study suggested that comorbidities had significant adverse effects on the prognosis of POTS in children. Therefore, we suggest that the comorbidity of pediatric POTS should be comprehensively evaluated during the diagnosis and treatment process, especially for children with recurrent symptoms and poor response to conventional treatment. In addition, for patients with comorbidities, individualized comprehensive therapies should be prescribed to obtain better therapeutic effects of POTS.

There are some limitations in our study. Due to the retrospective design of the study, some comorbidities may have been missed (such as hEDS/HSD) or underdiagnosed (such as migraine and sleeping disorder). And the sample size of this study was not very large. Anyway, based on the above research results, we conclude that comorbidity is an independent risk factor for CSRR in children with POTS, which not only significantly affects the prognosis but also aggravates the rehospitalization burden in children with POTS. Remarkably, more than one-third of pediatric POTS cases are complicated with allergic disorders. The relationship between pediatric POTS and comorbidities needs to be clarified in the future.

\section{Acknowledgments}

We would like to thank the foundation of the Peking University Clinical Scientist Program (BMU2019 LCKXJ001), and thanks to the Department of Pediatrics, Peking University First Hospital and all the researchers who contributed to this paper.

\section{Disclosure}

The authors report no conflict of interest in this work.

\section{References}

1. Wang YY, Du JB, Jin HF. Differential diagnosis of vasovagal syncope and postural tachycardia syndrome in children. World J Pediatr. 2020;16(6):549-552. doi:10.1007/s12519-019-00333-4

2. Hall J, Bourne KM, Sheldon RS, et al. A comparison of health-related quality of life in autonomic disorders: postural tachycardia syndrome versus vasovagal syncope. Clin Auton Res. 2021;31 (3):433-441. doi:10.1007/s10286-021-00781-x

3. Bhatia R, Kizilbash SJ, Ahrens SP, et al. Outcomes of adolescent-onset postural orthostatic tachycardia syndrome. J Pediatr. 2016;173:149-153. doi:10.1016/j.jpeds.2016.02.035

4. Tao C, Lu W, Lin J, et al. Long-term outcomes of children and adolescents with postural tachycardia syndrome after conventional treatment. Front Pediatr. 2019;7:261. doi:10.3389/fped.2019.00261

5. Liao Y, Zhang Q, Li H, Wang Y, Liu P, Du J. Co-morbidity of vasovagal syncope and postural tachycardia syndrome with allergic diseases in children. Beijing Da Yi Xue Bao Yi Xue Ban. 2017;49:41-46.

6. Reynolds GK, Lewis DP, Richardson AM, Lidbury BA. Comorbidity of postural orthostatic tachycardia syndrome and chronic fatigue syndrome in an Australian cohort. J Intern Med. 2014;275 (4):409-417. doi:10.1111/joim.12161

7. Miller AJ, Stiles LE, Sheehan T, et al. Prevalence of hypermobile Ehlers-Danlos syndrome in postural orthostatic tachycardia syndrome. Auton Neurosci. 2020;224:102637. doi:10.1016/j. autneu.2020.102637

8. Tu Y, Abell TL, Raj SR, Mar PL. Mechanisms and management of gastrointestinal symptoms in postural orthostatic tachycardia syndrome. Neurogastroenterol Motil. 2020;32(12):e14031. doi: $10.1111 / \mathrm{nmo} .14031$

9. McTate EA, Weiss KE. Psychosocial dimensions and functioning in youth with postural orthostatic tachycardia syndrome. Clin Pediatr (Phila). 2016;55(10):979-982. doi:10.1177/0009922815616890

10. Stewart JM, Boris JR, Chelimsky G, et al. Pediatric disorders of orthostatic intolerance. Pediatrics. 2018;141(1):e20171673. doi:10.1542/peds.2017-1673

11. Yun DJ, Choi HN, Oh GS. A case of postural orthostatic tachycardia syndrome associated with migraine and fibromyalgia. Korean J Pain. 2013;26(3):303-306. doi:10.3344/kjp.2013.26.3.303

12. Stewart JM, Medow MS, Cherniack NS, Natelson BH. Postural hypocapnic hyperventilation is associated with enhanced peripheral vasoconstriction in postural tachycardia syndrome with normal supine blood flow. Am J Physiol Heart Circ Physiol. 2006;291(2): H904-913. doi:10.1152/ajpheart.01359.2005

13. Roma M, Marden CL, De Wandele I, Francomano CA, Rowe PC. Postural tachycardia syndrome and other forms of orthostatic intolerance in Ehlers-Danlos syndrome. Auton Neurosci. 2018;215:89-96. doi:10.1016/j.autneu.2018.02.006

14. Ojha A, Chelimsky TC, Chelimsky G. Comorbidities in pediatric patients with postural orthostatic tachycardia syndrome. J Pediatr. 2011;158:20-23. doi:10.1016/j.jpeds.2010.07.005

15. Safder S, Chelimsky TC, O'Riordan MA, Chelimsky G. Gastric electrical activity becomes abnormal in the upright position in patients with postural tachycardia syndrome. J Pediatr Gastroenterol Nutr. 2010;51 (3):314-318. doi:10.1097/MPG.0b013e3181d13623

16. Wang Y, Du J, Jin H, Liao Y. Comorbidity of neurally mediated syncope and allergic disease in children. Front Immunol. 2020;11:1865. doi:10.3389/fimmu.2020.01865

17. Wang C, Li Y, Liao Y, et al. 2018 Chinese Pediatric Cardiology Society (CPCS) guideline for diagnosis and treatment of syncope in children and adolescents. Sci Bull. 2018;63:1558-1564. doi:10.1016/ j.scib.2018.09.019

18. Wang Y, Zhang C, Chen S, et al. Heart rate variability predicts therapeutic response to metoprolol in children with postural tachycardia syndrome. Front Neurosci. 2019;13:1214. doi:10.3389/ fnins.2019.01214 
19. Editorial Board, Chinese Journal of Pediatrics; Society of Pediatrics, Chinese Medical Association. Consensus on diagnosis and management of allergic diseases in children. Zhonghua $\mathrm{Er} \mathrm{Ke} \mathrm{Za} \mathrm{Zhi.}$ 2019;57(3):164-171.

20. Headache Classification Subcommittee of the International Headache Society. The international classification of headache disorders: 2nd edition. Cephalalgia. 2004;24(Suppl 1):9-160.

21. Rasquin A, Di Lorenzo C, Forbes D, et al. Childhood functional gastrointestinal disorders: child/adolescent. Gastroenterology. 2006;130(5):1527-1537. doi:10.1053/j.gastro.2005.08.063

22. Fukuda K, Straus SE, Hickie I, Sharpe MC, Dobbins JG, Komaroff A. The chronic fatigue syndrome: a comprehensive approach to its definition and study. International Chronic Fatigue Syndrome Study Group. Ann Intern Med. 1994;121(12):953-959. doi:10.7326/0003-4819-121-12-199412150-00009

23. Grahame R, Bird HA, Child A. The revised (Brighton 1998) criteria for the diagnosis of benign joint hypermobility syndrome (BJHS). $J$ Rheumatol. 2000;27(7):1777-1779.

24. Guo L, Zheng Y. Child and Adolescent Psychiatry. 2th ed. Beijing: People's Medical Publishing House; 2016.

25. Wolfe F, Clauw DJ, Fitzcharles MA, et al. The American College of Rheumatology preliminary diagnostic criteria for fibromyalgia and measurement of symptom severity. Arthritis Care Res. 2010;62 (5):600-610. doi:10.1002/acr.20140

26. Liu H, Zhong W, Zheng R. Clinical analysis of 28 cases with hyperventilation syndrome. China Modern Doctor. 2007;45 (22):39,65.

27. Malfait F, Francomano C, Byers P, et al. The 2017 international classification of the Ehlers-Danlos syndromes. Am J Med Genet C Semin Med Genet. 2017;175(1):8-26. doi:10.1002/ajmg.c.31552

28. Shaw BH, Stiles LE, Bourne K, et al. The face of postural tachycardia syndrome-insights from a large cross-sectional online community-based survey. J Intern Med. 2019;286(4):438-448. doi: $10.1111 /$ joim. 12895

29. Chelimsky G, Kovacic K, Nugent M, Mueller A, Simpson P, Chelimsky TC. Comorbid conditions do not differ in children and young adults with functional disorders with or without postural tachycardia syndrome. $J \quad$ Pediatr. 2015;167(1):120-124. doi:10.1016/j.jpeds.2015.03.039

30. Liao Y, Du J. Pathophysiology and individualized management of vasovagal syncope and postural tachycardia syndrome in children and adolescents: an update. Neurosci Bull. 2020;36(6):667-681. doi:10.1007/s12264-020-00497-4

31. Afrin LB, Ackerley MB, Bluestein LS, et al. Diagnosis of mast cell activation syndrome: a global "consensus-2". Diagnosis. 2020;8 (2):137-152. doi:10.1515/dx-2020-0005

32. Kohno R, Cannom DS, Olshansky B, et al. Mast cell activation disorder and postural orthostatic tachycardia syndrome: a clinical association. J Am Heart Assoc. 2021;10(17):e021002. doi:10.1161/ JAHA.121.021002

33. Kohn A, Chang C. The relationship between hypermobile Ehlers-Danlos Syndrome (hEDS), postural orthostatic tachycardia syndrome (POTS), and mast cell activation syndrome (MCAS). Clin Rev Allergy Immunol. 2020;58(3):273-297. doi:10.1007/s12016-01908755-8

International Journal of General Medicine

\section{Publish your work in this journal}

The International Journal of General Medicine is an international, peer-reviewed open-access journal that focuses on general and internal medicine, pathogenesis, epidemiology, diagnosis, monitoring and treatment protocols. The journal is characterized by the rapid reporting of reviews, original research and clinical studies
34. Rafael BS, Kelly YK, Pedro GB, Aun MV. Association of postural tachycardia syndrome and Ehlers-Danlos Syndrome with mast cell activation disorders. Immunol Allergy Clin North Am. 2018;38 (3):497-504. doi:10.1016/j.iac.2018.04.004

35. Piovesan EJ, Sobreira CF, Scola RH, et al. Episodic migraine associated with postural orthostatic tachycardia syndrome and vasovagal syncope: migraine triggers neuromediated syncope. Arq Neuropsiquiatr. 2008;66 (1):77-79. doi:10.1590/S0004-282X2008000100018

36. Folgering $\mathrm{H}$. The pathophysiology of hyperventilation syndrome. Monaldi Arch Chest Dis. 1999;54(4):365-372.

37. Lin J, Han Z, Li X, et al. Risk factors for postural tachycardia syndrome in children and adolescents. PLoS One. 2014;9(12): e113625. doi:10.1371/journal.pone. 0113625

38. Lin J, Zhao H, Shen J, Jiao F. Salivary cortisol levels predict therapeutic response to a sleep-promoting method in children with postural tachycardia syndrome. $J$ Pediatr. 2017;191:91-95.e1. doi:10.1016/j.jpeds.2017.08.039

39. Follenius M, Brandenberger G, Bandesapt JJ, Libert JP, Ehrhart J. Nocturnal cortisol release in relation to sleep structure. Sleep. 1992;15:21-27. doi:10.1093/sleep/15.1.21

40. Zipursky RT, Press MC, Srikanthan P, et al. Relation of stress hormones (urinary catecholamines/cortisol) to coronary artery calcium in men versus women (from the multi-ethnic study of atherosclerosis [Mesa]). Am J Cardiol. 2017;119(12):1963-1971. doi:10.1016/j. amjcard.2017.03.025

41. Okamoto LE, Raj SR, Peltier A, et al. Neurohumoral and haemodynamic profile in postural tachycardia and chronic fatigue syndromes. Clin Sci (Lond). 2012;122(4):183-192. doi:10.1042/CS20110200

42. Newton JL, Okonkwo O, Sutcliffe K, Seth A, Shin J, Jones DE. Symptoms of autonomic dysfunction in chronic fatigue syndrome. QJM. 2007;100(8):519-526. doi:10.1093/qjmed/hem057

43. Shoenfeld Y, Ryabkova VA, Scheibenbogen C, et al. Complex syndromes of chronic pain, fatigue and cognitive impairment linked to autoimmune dysautonomia and small fiber neuropathy. Clin Immunol. 2020;214:108384. doi:10.1016/j.clim.2020.108384

44. Celletti C, Borsellino B, Castori M, et al. A new insight on postural tachycardia syndrome in 102 adults with hypermobile Ehlers-Danlos Syndrome/hypermobility spectrum disorder. Monaldi Arch Chest Dis. 2020;90(2):1286. doi:10.4081/monaldi.2020.1286

45. Boris JR, Bernadzikowski T. Prevalence of joint hypermobility syndromes in pediatric postural orthostatic tachycardia syndrome. Auton Neurosci. 2021;231:102770.

46. Boris JR, Huang J, Shuey T, Bernadzikowski T. Family history of associated disorders in patients with postural tachycardia syndrome. Cardiol Young. 2020;30(3):388-394. doi:10.1017/S10479511 20000165

47. Stewart JM, Taneja I, Medow MS. Reduced body mass index is associated with increased angiotensin II in young women with postural tachycardia syndrome. Clin Sci (Lond). 2007;113(11):449-457. doi:10.1042/CS20070104

48. Li H, Wang Y, Liu P, et al. Body mass index (BMI) is associated with the therapeutic response to oral rehydration solution in children with postural tachycardia syndrome. Pediatr Cardiol. 2016;37 (7):1313-1318. doi:10.1007/s00246-016-1436-1

across all disease areas. The manuscript management system is completely online and includes a very quick and fair peer-review system, which is all easy to use. Visit http://www.dovepress.com/ testimonials.php to read real quotes from published authors. 